\title{
Alfabetización científica en la etapa 3-6 años: un análisis de la regulación estatal de enseñanzas mínimas
}

\section{Scientific literacy at the 3-6 year old stage: an analysis of Spain's national curriculum}

\author{
Antonio García-Carmona, Ana M. Criado, Pedro Cañal \\ Departamento de Didáctica de las Ciencias. Universidad de Sevilla \\ garcia-carmona@us.es, acriado@us.es,pcanal@us.es
}

RESUMEN • Se analiza la alfabetización científica promovida por la regulación de enseñanzas mínimas para la etapa 3-6 años en Espańa. Para ello, se diseñó y aplicó un cuestionario combinando procesos de análisis inter- e intra-jueces como estrategia de validación y fiabilidad. Se valoró cómo el documento atiende a las siguientes dimensiones, relativas a la ciencia escolar: construcción de la ciencia escolar; axiología y psicología del aprendizaje de la ciencia; objetivos y competencias; contenidos; actividades, diseño y estrategias de enseñanza; recursos didácticos, y evaluación. Los resultados indican que la ciencia escolar sugerida en el documento no sintoniza, en una parte importante, con las tendencias actuales de la Didáctica de las Ciencias. A consecuencia de las carencias detectadas, se hacen algunas recomendaciones que mejorarían la propuesta oficial.

PALABRAS CLAVE: alfabetización científica; ciencia escolar; enseñanza de la ciencia; educación infantil; regulación de enseñanzas mínimas.

ABSTRACT - An analysis is made of the scientific literacy promoted by Spain's national curriculum for the 3-6 year old stage. To this end, a questionnaire was designed and implemented combining inter- and intra-rater processes of analysis as a strategy of validation and reliability. An evaluation was made of how the official document addresses the following dimensions of science education at this stage: the construction of school science; the axiology and psychology of learning science; objectives and competences; content; teaching activities, design, and strategies; and teaching resources and evaluation. The results indicate that the school science actually being put forward in the document is, to a major degree, out of tune with current trends in Science Teaching. In view of the deficiencies identified in the document several improvements are recommended.

KEYWORDS: childhood education; national curriculum; school science; science education; scientific literacy.

Fecha de recepción: noviembre 2011 • Aceptado: marzo 2013

García-Carmona, A., Criado, A.M., Cañal, P. (2014) Alfabetización científica en la etapa 3-6 años: un análisis de la regulación estatal de enseñanzas mínimas. Enseñanza de las 


\section{INTRODUCCIÓN}

La alfabetización cientifica se erige hoy como una meta prioritaria dentro de la educación básica de todas las personas (Acevedo, 2004). Sin embargo, la consecución de tal propósito está todavía lejos de ser logrado en una parte importante de países. En el contexto europeo dan buena prueba de ello los informes de Rocard et al. (2007) y el de la fundación Nuffield (Osborne y Dillon, 2008).

En España, la cuestión es todavía más preocupante si cabe. El nivel de competencia científica de los alumnos españoles, según las últimas evaluaciones PISA, se sitúa por debajo de la media de los países de la OCDE. A consecuencia de ello, la Confederación de Sociedades Científicas de España (COSCE) ha publicado el informe ENCIENDE (2011), donde advierte de la urgente necesidad de impulsar una adecuada educación científica desde los 3 años de edad. Ello requiere, indefectiblemente, la atención y mejora de una diversidad de aspectos relativos a la ciencia escolar desde el 2..$^{\circ}$ ciclo de infantil (3-6 años). $Y$ es que tal etapa educativa es fundamental en la aproximación inicial de los escolares a las perspectivas científicas sobre la realidad (Cañal, 2006; Spektor-Levy, Kesner y Mevarech, 2013).

Los informes europeos aludidos señalan, principalmente, al profesorado y a diseñadores de materiales curriculares como máximos responsables de la insuficiente alfabetización científica de la ciudadanía, en general. El informe ENCIENDE añade, además, una crítica sobre el poco peso específico de la ciencia escolar, frente a otras áreas, en los niveles educativos obligatorios espańoles. Por lo demás, los tres informes proponen una serie de recomendaciones similares, orientadas a promover una alfabetización científica básica, que sea acorde con las exigencias de la sociedad actual.

Sin embargo, se echa de menos un análisis crítico de lo que realmente prescriben las autoridades educativas; esto es, una valoración de hasta qué punto las disposiciones de los currículos oficiales de ciencias contribuyen también a que la alfabetización científica no sea apropiadamente desarrollada en las aulas.

Son escasos los estudios que analizan directamente el currículo oficial para determinar sus puntos fuertes y débiles; y menos aún, referidos a la etapa de infantil. Si bien, los que se conocen en esta línea de investigación revelan carencias significativas en tales documentos prescriptivos. García de Cajén et al. (2002) encontraron que el currículo oficial argentino para primaria y secundaria no prescribe adecuadamente las estrategias de razonamiento y de argumentación más idóneas para el aprendizaje de las ciencias. En España, destacan los trabajos de De Pro y Miralles (2009) y Banet (2010) sobre la educación científica regulada en la regulación oficial LOE de enseñanzas mínimas para primaria. Analizan la incorporación de las competencias como elemento novedoso, así como la sintonía de los objetivos, contenidos y criterios de evaluación con las tendencias actuales en Didáctica de las Ciencias. El último de ellos examina, además, las orientaciones del currículo vigente para la educación científica, comparando sus novedades respecto a las prescripciones de las dos reformas educativas anteriores (LOGSE y LOCE). Ambos estudios detectan carencias relevantes, que pueden obstaculizar una educación científica primaria acorde con las exigencias socioculturales actuales. Ello lo hemos podido corroborar en un estudio más reciente (García-Carmona, Criado y Cañal, 2014), al detectar ciertas insuficiencias en las prescripciones de la LOE, que no favorecen la alfabetización científica esperada en la etapa de primara.

No obstante, nuestra alineación con la necesidad de promover la alfabetización científica desde los 3 años nos lleva a cuestionar también -como parte de un estudio más amplio- en qué medida la regulación española de enseñanzas mínimas para la etapa 3-6 años favorece u obstaculiza tal empeño. 


\section{PLANTEAMIENTO DE LA CUESTIÓN}

A la vista de lo expuesto, y dado que no conocemos estudios precedentes, nos planteamos investigar: ¿Qué sugerencias hace la regulación estatal de enseñanzas minimas para la educación infantil, con vistas a promover una alfabetización cientifica inicial, y hasta qué punto son coherentes con las tendencias actuales en Didáctica de las Ciencias?

Para ello, se analizó el documento oficial que regula las enseñanzas mínimas para la etapa 3-6 años, establecida por el Ministerio de Educación en el RD 1630/2006, de 29 de diciembre (<http://www. boe.es/boe/dias/2007/01/04/pdfs/A00474-00482.pdf>).

La investigación parte del supuesto de que el insuficiente nivel alcanzado por los escolares españoles en competencia científica no es solo consecuencia de malogradas implementaciones de los currículos oficiales en las aulas, sino que sus prescripciones también desempeñan un papel importante en ello. Esto es, se asume como hipótesis a contrastar que el documento oficial citado no prescribe una ciencia escolar totalmente acorde con las tendencias actuales en Didáctica de las Ciencias; bien por omisiones, por planteamientos incoherentes, o por alusiones excesivamente superficiales o poco clarificadoras. Los resultados de los estudios mencionados en la introducción, aunque se refieren a la etapa educativa adyacente, apoyan la consideración de esta hipótesis.

\section{PROCEDIMIENTO}

Para el análisis se diseñó un cuestionario (anexo) con diez dimensiones relativas a diferentes aspectos didácticos y epistemológicos de la ciencia escolar: (1) construcción de la ciencia escolar, (2) fundamentos axiológicos, (3) fundamentos psicológicos, (4) objetivos y competencias, (5) contenidos, (6) actividades, (7) estrategia de enseñanza, (8) recursos, (9) diseño de la enseñanza y (10) evaluación.

Se trató de verificar si el contenido de los indicadores de las dimensiones anteriores se incluye en el documento objeto de análisis, y, dado el caso, de qué modo. Es decir, se valoró si:

- Se indica o sugiere clara y explícitamente el aspecto.

- Se indica implicitamente el aspecto, de manera superficial o incompleta.

- No se indica o menciona el aspecto.

El diseño del cuestionario partió, por un lado, de las conclusiones de un estudio anterior sobre obstáculos y dificultades de los profesores de primaria en su práctica docente (Pozuelos, Travé y Cañal, 2010); y, por otro, de una revisión exhaustiva de la literatura en torno a las dimensiones indicadas -parte de esta se cita en la discusión de los resultados-. Las conclusiones de ese estudio anterior permitieron establecer los interrogantes guía para cada una de las dimensiones; y la revisión bibliográfica, una propuesta inicial de respuestas expertas a tales preguntas, a modo de referentes teóricos o estándares, para favorecer el análisis. Estos estándares fueron escogidos entre aquellos posicionamientos de la literatura que gozan de mayor consenso en la actualidad.

La aplicación del cuestionario se sometió a procesos de validación y fiabilidad, que combinaron estrategias de análisis inter- e intra-jueces (Padilla, 2002). Se partió de un primer borrador consensuado por dos investigadores del equipo, de acuerdo con lo expuesto antes. Este fue revisado por un tercer investigador del equipo e hizo sus aportaciones para obtener una segunda versión. Con esta, los dos investigadores iniciales realizaron independientemente un análisis preliminar completo del documento, con posterior puesta en común de los resultados (análisis interjueces). De este análisis preliminar surgió una versión mejorada y definitiva (incluida en el anexo). Cuatro meses después, uno de los investigadores volvió a realizar el mismo análisis con la versión final (análisis intrajuez), obteniendo resultados más significativos que en el preliminar, y que son los que aquí se presentan. 


\section{RESULTADOS}

Presentamos los resultados con el agrupamiento de algunas de las dimensiones analizadas por su proximidad o relación, a fin de facilitar su presentación y discusión.

\section{Construcción de la ciencia escolar}

La ciencia académica debe ser el referente para determinar la alfabetización científica deseable, pero su adecuación al ámbito escolar debe venir dada por una transformación, o transposición didáctica de los contenidos, que responda a las características psicocognitivas de los escolares y a las finalidades educativas de la etapa en cuestión. Surge así la ciencia escolar, cuya finalidad es, a grandes rasgos, proveer a los escolares de unas competencias básicas que les permitan entender -con cierta aproximación-el mundo natural y tecnológico en el que viven, interaccionar con él adecuadamente, y valorar con sentido crítico las múltiples causas y consecuencias del desarrollo científico-tecnológico.

La transposición didáctica de la ciencia al ámbito educativo no es una tarea sencilla, dada la diversidad de factores que se deben tener en cuenta. Sin embargo, el proceso permite planificar una ciencia adecuada a los intereses, capacidades y experiencias de los escolares. En los niveles elementales, los escolares poseen una visión egocéntrica y sincrética del medio natural, lo que desaconseja promover una enseñanza formal de la ciencia (Marín, 2005). Se recomienda, entonces, comenzar fomentando la curiosidad por fenómenos naturales sencillos, que sean fácilmente perceptibles e investigables por los escolares en su entorno más inmediato (Cañal, 2006; Spektor-Levy, Kesner y Mevarech, 2013). Para ello, es esencial partir de sus concepciones, considerando que estas no son erróneas sino, más bien, ideas incompletas o distintas a las aceptadas por la ciencia. Y para que tales ideas evolucionen hacia el conocimiento científico escolar deseable, habrá que ayudar a los escolares a que las pongan en juego y valoren si son útiles o deben modificarlas, con vistas a poder explicar la realidad que los rodea (Tonucci, 1995).

El documento que se analiza sugiere las ideas anteriores, sintonizando así con una visión actualizada de la Didáctica de las Ciencias. Por ejemplo, dice:

Las interacciones que nińas y niños establezcan con los elementos del medio, (...) deben constituir situaciones privilegiadas que los llevarán a crecer, a ampliar sus conocimientos sobre el mundo y a desarrollar habilidades, destrezas y competencias nuevas. (p. 478)

\section{Fundamentos axiológicos}

La ciencia escolar debe impulsar entre los escolares el desarrollo de una serie actitudes y valores que los ayuden a analizar y participar, como ciudadanos críticos y responsables, ante situaciones sociocientíficas. La etapa 3-6 años debe ser donde se fragüen los primeros cimientos para el desarrollo progresivo de tal competencia, centrando la atención en aquellos aspectos o situaciones más cercanas y palpables para los niños; por ejemplo, la iniciación a la reflexión crítica y la toma de decisiones relativas a hábitos de cuidado y respeto por la conservación del entorno más próximo; el respeto por los animales; los hábitos de higiene y una alimentación saludable, etc. (Marín, 2005; Cañal, 2006). Ello se sugiere en el documento como sigue:

(...) el desarrollo de las destrezas y capacidades individuales y su interacción con el medio y con los iguales contribuyen a la evolución del pensamiento, enseñando a pensar y a aprender (pensamiento crítico, toma de decisiones, resolución de problemas, etc.) (...). (p. 476) 
La apreciación de la diversidad y riqueza del medio natural, el descubrimiento de que las personas formamos parte de ese medio, la vinculación afectiva al mismo, son la base para fomentar desde la escuela actitudes habituales de respeto y cuidado. (p. 478)

\section{Fundamentos psicológicos}

Ya hemos avanzado la importancia de los conocimientos y experiencias previas de los escolares en la construcción de conocimiento científico escolar. Esto se recoge en el documento como sigue:

Las vivencias que tienen en relación con los elementos de la naturaleza y la reflexión sobre ellas, los llevarán, con el apoyo adecuado de la escuela, a la observación de algunos fenómenos naturales, sus manifestaciones y consecuencias, así como a acercarse gradualmente al conocimiento de los seres vivos (...). (p. 478)

Sin embargo, el aprendizaje de la ciencia, en cualquier nivel educativo, tiene unas características específicas y diferenciadas respecto a otras áreas (Pozo y Gómez, 1998), que merecen cierta atención en las prescripciones oficiales. Es insuficiente que el documento oficial limite su aportación, al respecto, aduciendo simplemente "con el apoyo adecuado de la escuela". Debería subrayarse que las vivencias y los conocimientos cotidianos de los escolares son el punto de partida idóneo para orientar la adquisición de los nuevos conocimientos, mediante una propuesta de actividades que los ayude a tomar conciencia de las limitaciones de sus ideas intuitivas, y de cómo otros conocimientos escolares pueden ser más efectivos para comprender los fenómenos. Refiriéndose a ello, y para la etapa de infantil, Cañal (2006) destaca que:

La riqueza y la validez de los conocimientos del niño sobre su entorno guardan una estrecha relación con la frecuencia, calidad y diversidad de las experiencias que haya vivido, ya que estas le proporcionan la base empírica que le resulta necesaria para el desarrollo de sus instrumentos conceptuales y metodológicos, y también para el fortalecimiento de las actitudes y motivaciones que impulsan estos procesos cognitivos. (p. 6)

Esto último debe ir acompañado de la toma de conciencia de las potencialidades y carencias propias, así como de voluntad y perseverancia para adquirir nuevos aprendizajes; aspectos que tampoco se mencionan en el documento y que conviene ir fraguando desde la etapa educativa inicial.

El documento sí habla de los factores psicocognitivos y afectivos que influyen en el aprendizaje, posicionándose explícita y convenientemente como sigue:

Cada niño tiene su ritmo y su estilo de maduración, desarrollo y aprendizaje, por ello, su afectividad, sus características personales, sus necesidades, intereses y estilo cognitivo, deberán ser también elementos que condicionen la práctica educativa en esta etapa. (p. 478)

También se refiere, como se ha citado antes, al papel que desempeñan la curiosidad y los intereses de los escolares en el aprendizaje de la ciencia escolar. Y resalta, como cuestión trasversal esencial para el aprendizaje de cualquier materia, el desarrollo de habilidades socioafectivas de los escolares entre las principales metas educativas de la etapa de infantil. Concretamente, dice:

El currículo se orienta a lograr un desarrollo integral y armónico de la persona en los distintos planos: físico, motórico, emocional, afectivo, social y cognitivo, y a procurar los aprendizajes que contribuyen y hacen posible dicho desarrollo. (p. 474)

Del mismo modo, el documento hace claras alusiones a la importancia de propiciar un buen clima de aula durante el proceso de aprendizaje, resaltando su incidencia en aspectos como la autoestima, la autonomía, la integración social y la responsabilidad. Ejemplos de estas alusiones: 
Los métodos de trabajo (...) se aplicarán en un ambiente de afecto y confianza, para potenciar su autoestima e integración social. (p. 474)

(...) realizar las actividades habituales con un cierto grado de responsabilidad, autonomía e iniciativa en la utilización adecuada de espacios y materiales, y en el desempeño de las diversas tareas que se realizan en el aula. (p. 476)

En cambio, el documento no es nada explícito sobre la importancia de la interacción comunicativa durante el aprendizaje. Aun cuando se refiere a la necesidad de iniciar a los niños en la colaboración, no justifica su ventaja educativa; tampoco se refiere a la importancia del aprendizaje cooperativo, en general, y particularmente en el caso de la ciencia escolar. Lo más que dice es:

Adecuar su comportamiento a las necesidades y requerimientos de los otros, desarrollando actitudes y hábitos de respeto, ayuda y colaboración, evitando comportamientos de sumisión o dominio. (p. 477)

Habilidades para la interacción y colaboración y actitud positiva para establecer relaciones de afecto con las personas adultas y con los iguales. (p. 477)

Si la regulación oficial no atiende adecuadamente tal cuestión, en poco ayudará a evitar que la escuela infantil española aún fomente relativamente poco el trabajo cooperativo (Lera, 2006). El enfoque socioconstructivista es idóneo para el aprendizaje de las ciencias (Furió y Furió, 2009), y sugiere la construcción de aprendizajes en un plano de similitud cognitiva; de modo que la meta debe ser que los escolares conciban el aprendizaje como el conjunto de conclusiones a las que se llega colectivamente, con la ayuda del profesor, después de observar, plantear preguntas, planificar, experimentar, analizar, comprobar y valorar posibles explicaciones sobre las cuestiones abordadas.

\section{Objetivos y competencias}

Además de la componente actitudinal, el documento propone entre sus objetivos el desarrollo de destrezas básicas para una alfabetización científica inicial. Concretamente, la adquisición de capacidades propias de la indagación científica (escolar), a saber:

Observar y explorar de forma activa su entorno, generando interpretaciones sobre algunas situaciones y hechos significativos, y mostrando interés por su conocimiento. (p. 479)

En infantil se trata, efectivamente, de comenzar con el fomento de la curiosidad de los escolares por los fenómenos naturales sencillos de su alrededor. Ello favorecerá la construcción de unos primeros conocimientos que les permitan comprender y actuar responsablemente en el medio natural. En este sentido, también establece entre sus objetivos la adquisición de conocimientos o interpretaciones de los fenómenos y objetos físicos de su entorno:

Conocer y valorar los componentes básicos del medio natural y algunas de sus relaciones, cambios y transformaciones (...). (p. 479)

Por otra parte, el documento insinúa, de manera más bien implícita, que los objetivos de aprendizaje deben concebirse como unos referentes flexibles y adaptables a cada situación educativa. Hace alusión a ello en el apartado Atención a la Diversidad, diciendo que:

Los centros atenderán a los nińos y niñas que presenten necesidades educativas especiales buscando la respuesta educativa que mejor se adapte a sus características y necesidades personales. (p. 475)

Y en el apartado Autonomía de los Centros, estableciendo que: 
Los centros docentes desarrollarán y completarán el currículo establecido por las administraciones educativas adaptándolo a las características de los nińos y niñas y a su realidad educativa. (p.475).

En relación con las competencias básicas, y a diferencia del currículo oficial de primaria, el de infantil no incluye su desarrollo entre las metas educativas de la etapa. Sugiere, razonablemente, que de 3 a 6 ańos se debe aspirar a sentar las primeras bases para su posterior y progresivo desarrollo en las etapas subsiguientes:

En esta etapa educativa se sientan las bases para el desarrollo personal y social y se integran aprendizajes que están en la base del posterior desarrollo de competencias que se consideran básicas para todo el alumnado. (p. 476)

En ese marco, el documento aporta sugerencias explícitas y genéricas sobre cómo puede contribuirse a esa primera cimentación de las diferentes competencias. En el caso específico de las áreas del currículo que pueden propiciar escenarios de alfabetización científica, además de la propia competencia científica $-\mathrm{y}$ de otras genéricas ya citas- se destaca explícitamente su idoneidad para iniciar el desarrollo de las competencias comunicativa, matemática, cultural y la digital o tecnológica (p. 478):

(...) el entorno no puede ser comprendido sin la utilización de los diferentes lenguajes (...).

Para conocer y comprender cómo funciona la realidad, el niño (...) detecta semejanzas y diferencias, compara, ordena, cuantifica, pasando así de la manipulación a la representación, origen de las incipientes habilidades lógico matemáticas.

El entorno infantil debe ser entendido (...) como el espacio de vida que rodea a niños y niñas, en el que se incluye lo que afecta a cada uno individualmente y lo que afecta a los diferentes colectivos de pertenencia (...). Así, las niñas y los niños reconocerán en ellos las dimensiones física, natural, social y cultural que componen el medio en que vivimos.

La importancia de las tecnologías como parte de los elementos del entorno aconseja que niñas y niños identifiquen el papel que estas tecnologías tienen en sus vidas, interesándose por su conocimiento e iniciándose en su uso.

No obstante, en el documento se echa en falta cierta atención a la competencia relacionada con la socialización y la afectividad, dentro de sus sugerencias para el área de conocimiento del entorno (solo se refiere a ello de un modo genérico, como indicábamos más arriba en los resultados de la dimensión "fundamentos psicológicos"). Se limita a decir que la etapa debe contribuir a sentar las bases para la socialización de los escolares (p. 478), pero no incide en el papel que puede tener la educación científica en el desarrollo social y emotivo de los escolares de cualquier nivel educativo (Garritz, 2009), y especialmente de la etapa de infantil (Spektor-Levy, Kesner y Mevarech, 2013); por ejemplo, mediante la promoción de dinámicas de trabajo en equipo con talleres de ciencia recreativa, donde los escolares puedan disfrutar con la ciencia; o a través de talleres de diseño y construcción de juguetes con material reciclado (marionetas, cochecitos de cartón, etc.), a fin de que empiecen a desarrollar un pensamiento tecnológico, en un clima lúdico y afectivo, a la vez que potencien su capacidad manipulativa.

Igualmente se desatiende la competencia para aprender a aprender, aun cuando ya se viene aconsejando su promoción desde infantil (Martín, 2008). Como adelantábamos, en esta etapa podría iniciarse con el fomento de actitudes de voluntad y perseverancia en el aprendizaje. Es conveniente virar la habitual ansiedad de los niños por recibir respuestas inmediatas sobre los fenómenos naturales que observan, hacia una actitud que asuma la comprensión de la naturaleza como un proceso pautado y progresivo, que depende también de la propia indagación de los escolares. De ahí que se sugiera para las primeras etapas educativas centrar la atención en cómo aprender ciencia, más que en qué ciencia aprender. 


\section{Contenidos}

Los contenidos deben ser considerados como los medios, escenarios o informaciones, de tipo conceptual, procedimental y actitudinal, que se ponen en juego para que los escolares aproximen sus conocimientos progresivamente a lo establecido en los objetivos. Asimismo, los distintos tipos de contenidos deben plantearse de un modo relacionado y no fragmentado. La regulación de enseñanzas mínimas de infantil favorece esa visión de los contenidos con argumentaciones como las siguientes:

Estas áreas deben entenderse como ámbitos de actuación, como espacios de aprendizajes de todo orden: De actitudes, procedimientos y conceptos, que contribuirán al desarrollo de niñas y niños y propiciarán su aproximación a la interpretación del mundo (...). (p. 475)

Los contenidos (...) adquieren sentido desde la complementariedad con el resto de las áreas, y habrán de interpretarse en las propuestas didácticas desde la globalidad de la acción y de los aprendizajes. (p. 478)

Asimismo, el documento provee una relación coherente entre los objetivos de aprendizaje y los contenidos propuestos para el desarrollo de una alfabetización científica inicial, en la línea que venimos argumentando. Sin embargo, no se da ninguna orientación -aunque sea a modo de hipótesis- sobre la progresión con que deberían introducirse tales contenidos a lo largo de la etapa. Aun sin dejar de reconocer que es una cuestión compleja y que, por tanto, no existiría un único planteamiento, ello supondría un primer referente para que el profesorado organice su enseñanza (Prieto, Blanco y Brero, 2002). Lógicamente, tal referente debe ser luego matizado y afinado de acuerdo con las características de cada contexto educativo. Una propuesta de progresión de índole procedimental podría ser: primero, aprender a observar, de forma organizada, objetos y fenómenos; segundo, aprender a verbalizar lo observado; tercero, incrementar paulatinamente el número de aspectos a observar en torno a un mismo fenómeno, e igualmente verbalizar lo observado; y cuarto, lograr una primera transición de la descripción a la explicación de lo observado.

\section{Actividades, recursos y estrategias de enseñanza}

El documento se refiere a las actividades en reiteradas ocasiones cuando da alguna orientación sobre estrategias de enseñanza; sin embargo, no entra a definirlas ni a sugerir criterios orientadores, que ayuden a establecer secuencias lógicas de estas. Solo dice que:

Los contenidos educativos de la Educación infantil se (...) abordarán por medio de actividades globalizadas que tengan interés y significado para los nińos. (p. 474).

No olvidemos que el desarrollo último del currículo se lleva a cabo mediante los programas de actividades que se diseñan. En consecuencia, debería dedicársele cierta atención describiendo qué son, qué tipos pueden plantearse, según los recursos y finalidades educativas que tengan, etc. Aunque no existe una única clasificación de actividades, todas las propuestas parten de la idea de que una actividad es una acción educativa que favorece el proceso de enseñanza-aprendizaje (Fernández et al., 2002). Su alusión en la regulación de enseñanzas mínimas ayudaría a diluir la concepción sesgada y simplista que suele tenerse de las actividades en la enseñanza, llegándose a identificar, muchas veces, con la realización mecánica de simples ejercicios en el aula; entre ellos, el sistema de fichas aún muy extendido en esta etapa educativa (Lera, 2006). Un ejemplo de esquema de clasificación de actividades, para el desarrollo del aprendizaje por indagación en infantil es el propuesto por Cañal (2006).

Asimismo, aunque el documento habla de la importancia de indagar sobre el medio como vía de aprendizaje, omite elementos esenciales de este modelo didáctico, como: la selección de los objetos de estudio; la formulación de los problemas a indagar; la participación de los escolares en dicha formula- 
ción; la planificación de la investigación; la selección y el registro de la información, etc. (Cañal, 2006). Por tanto, menos aún sugiere cuáles de ellos serían recomendables introducir al inicio de la etapa, y con qué posible progresión iría integrándose el resto hasta conformar una iniciación a la investigación escolar en infantil. El documento se limita a decir:

Para conocer y comprender cómo funciona la realidad, el niño indaga sobre el comportamiento y las propiedades de objetos y materias presentes en su entorno: actúa y establece relaciones con los elementos del medio físico, explora e identifica dichos elementos, reconoce las sensaciones que producen, se anticipa a los efectos de sus acciones sobre ellos, detecta semejanzas y diferencias, compara, ordena, cuantifica (...). (p. 478)

Respecto a las fuentes de contenidos y recursos didácticos para iniciar la alfabetización científica en infantil, el currículo se refiere, en todo momento y de una manera genérica, al medio socionatural y sus componentes:

Se concibe, pues, el medio como la realidad en la que se aprende y sobre la que se aprende. (p. 478)

El medio natural y los seres y elementos que lo integran se convierten bien pronto en objetos preferentes de la curiosidad e interés infantil. (p. 478)

Sin embargo, el espacio habitual de aprendizaje de los escolares será el aula y demás dependencias del centro educativo, de modo que estaría bien que se sugirieran recursos y estrategias concretas para promover la alfabetización científica en dicho contexto. Como ya hemos comentado, se podría destacar, al respecto, la propuesta de rincones o talleres de experiencias científicas en el aula (Criado y García-Carmona, 2011; Sánchez et al., 2008, etc.), o la creación y cuidado de huertos en el centro escolar (Roás, 2001), entre otros. De la misma manera, y como complemento a lo anterior, sería interesante resaltar las visitas extraescolares a museos y exposiciones científicas, donde cada vez se dedica mayor atención al público infantil (De Pablo, 2006).

Por otra parte, como se adelantó, el documento sugiere la iniciación al uso de las TIC como recurso de apoyo en el aprendizaje sobre el medio.

\section{Diseño de la enseńanza}

Hemos dicho que el documento insta a que los centros educativos sean los que concreten las enseñanzas prescritas a su realidad socioeducativa. Sin embargo, en tal marco debería sugerir también que el diseño de las propuestas didácticas, adaptadas a cada contexto educativo, debería corresponder al profesorado. Esto se viene demandando hace ya tiempo en el ámbito de la Didáctica de las Ciencias, con planteamientos y enfoques muy diversos para las primeras etapas de la educación (Pasek et al., 2010; Romero, 2011; Varela y Plasencia, 2006, etc.). Su desatención en la regulación oficial de enseñanzas mínimas no contribuye a superar el hecho de que las editoriales sean las que finalmente determinan, en muchos casos, qué se va a enseñar en las aulas y con qué nivel (De Pro et al., 2008). No promovemos, con esto, el rechazo hacia los libros de texto y sus cuadernillos o fichas correspondientes, sino que se conciban como un recurso didáctico más, entre otros. Si bien, es cierto que todo ello requiere una adecuada formación científico-didáctica del profesorado de infantil (Rodríguez et al., 2007), cuya disposición traspasa, lógicamente, los límites del documento analizado.

\section{Evaluación}

Al igual que en el aprendizaje de cualquier área, la evaluación tiene un papel fundamental en la educación científica. Las alusiones del documento a la evaluación se hacen desde una perspectiva genérica 
y para el conjunto de áreas que componen la etapa. Solo los criterios de evaluación se particularizan para cada una de las áreas de conocimiento. Los criterios que se proponen para valorar la alfabetización científica esperada en la etapa pueden considerarse adecuados: por un lado, porque guardan una relación clara y coherente con los objetivos y contenidos propuestos para dicha alfabetización; y, por otro, porque el nivel de aprendizaje exigido es coherente con las sugerencias de la literatura actual al respecto. Sin embargo, tal y como argumentábamos para los contenidos, y asumiendo lo que el propio documento indica sobre el papel de los criterios de evaluación (“...se conciben como una referencia para orientar la acción educativa”, p. 474), hubiese sido deseable una propuesta desglosada y progresiva de estos -a modo de hipótesis orientadoras-, hasta llegar al nivel máximo estimado al finalizar la etapa.

Las sugerencias del documento sobre la evaluación son, en general, bastante acordes con las actuales tendencias en evaluación educativa (Castillo y Cabrerizo, 2003). Esto es, se hace hincapié en su dimensión pedagógica o formativa (“...la evaluación debiera tener como fin la identificación de los aprendizajes adquiridos así como la valoración del desarrollo alcanzado teniendo, por tanto un carácter netamente formativo", p. 474); se defiende su carácter globalizador para esta etapa educativa; y se incide en que también debe evaluarse la práctica docente ("Los maestros que impartan el segundo ciclo de la Educación infantil evaluarán, además de los procesos de aprendizaje, su propia práctica educativa”, p. 475). Pero no se refiere a la evaluación formadora, es decir, la que implica a los escolares en su propia evaluación. Tal dimensión evaluativa guarda estrecha relación con la competencia para aprender a aprender, cuyo desarrollo, como hemos dicho, convendría iniciar en esta etapa.

En cuanto a los instrumentos de evaluación, el documento da prioridad a la observación sistemática en clase ("La observación directa y sistemática constituirá la técnica principal del proceso de evaluación”, p. 475). Este posicionamiento puede resultar comprensible para la etapa de infantil, pero también es razonable exigir que el documento haga referencia a otros instrumentos complementarios para el enriquecimiento del proceso evaluativo; por ejemplo, el uso del portafolio.

\section{CONCLUSIONES Y PROPUESTAS DE MEJORA}

El análisis realizado permite concluir que la regulación española de enseñanzas mínimas para la etapa 3-6 años promueve una alfabetización científica inicial que concuerda, en muchos de sus planteamientos, con los posicionamientos actuales sobre educación científica elemental. Sin embargo, se detectan también aspectos esenciales de esta que no son atendidos, o lo son de manera inadecuada. Es cierto que el documento analizado es genérico y abierto, pero si da orientaciones didácticas y metodológicas para la enseñanza, parece oportuno demandarle que incida adecuadamente en aquellos aspectos sugeridos por las actuales tendencias en Didáctica de las Ciencias.

De las carencias detectadas surgen las recomendaciones siguientes, como propuestas que mejorarían la alfabetización científica prescrita desde la regulación de enseñanzas mínimas para la etapa de infantil:

- Debería incidirse en que las ideas y experiencias previas de los escolares deben constituir el punto de partida para orientar el proceso de aprendizaje; y que la interacción comunicativa y permanente en un plano de similitud cognitiva (bajo las orientaciones del profesor) favorece el aprendizaje de la ciencia.

- Si bien el documento hace alusión explícita al desarrollo de ciertas competencias básicas como la comunicativa, matemática, cultural y la digital, desde el área de conocimiento del entorno, sería recomendable que el documento hiciera una apuesta más amplia y decidida en torno a una educación científica basada en el desarrollo de competencias. Así, debería mostrar una atención especial a las competencias que tienen que ver con la socialización y la afectividad de los 
escolares, ampliamente promulgadas desde la literatura actual en pos de una mejor educación científica básica. En este sentido, habría que aprovechar las oportunidades que ofrecen los talleres de ciencia recreativa para producir emociones positivas, a la vez que se aprende; o los talleres de fabricación de sencillos artefactos, que permiten iniciar el pensamiento tecnológico en un clima socioafectivo y lúdico. Del mismo modo, debería prestarse atención a la competencia para aprender a aprender, fomentando la curiosidad, la voluntad, la paciencia y la perseverancia como actitudes esenciales para lograr un primer conocimiento de la naturaleza circundante.

- Sería aconsejable ofrecer alguna orientación para la introducción y el desarrollo progresivo de los conocimientos escolares de ciencias a lo largo de la etapa, así como para la propuesta de los criterios de evaluación correspondientes.

- Debería prestarse atención a las actividades de enseñanza-aprendizaje, ofreciendo algunas orientaciones básicas sobre su diseño, secuenciación, finalidades didácticas, etc.

- Como se promueve una estrategia de aprendizaje de la ciencia basada en la investigación escolar, debería incidir adecuadamente en las características y los planteamientos esenciales de tal modelo didáctico.

- Más allá de citar al medio como escenario para el aprendizaje de la ciencia escolar inicial, debería hacerse referencia explícita a una diversidad de recursos específicos que permiten su desarrollo en el aula (por ejemplo, rincones o talleres de ciencias) y fuera de ella (exposiciones científicas, huertos, etc.).

- Debería impulsarse que el profesorado diseñe o adapte su propio material didáctico para el desarrollo de su enseñanza, promoviendo no solo los recursos didácticos y metodológicos del punto anterior, sino también la integración paulatina de materiales e instrumentos que la sociedad utiliza para comprender el mundo natural (balanzas, sensores, instrumentos ópticos, documentales, libros, ordenadores, etc.).

- Debería prestarse atención a la dimensión formadora de la evaluación, que adjudica a los escolares responsabilidad en ella.

- Debería hacerse alusión a una variedad de instrumentos para la evaluación de los aprendizajes, y no limitarse a la observación directa en el aula.

Finalmente, hemos de decir que somos conscientes de que la inclusión de sugerencias como las anteriores, en un documento oficial como el analizado, no implica que luego sean asumidas por el profesorado en su práctica. Pero también estamos convencidos de que si no se integran en los documentos marco, muy posiblemente no sean concebidas como esenciales y, por tanto, su incidencia en las aulas será imperceptible.

\section{AGRADECIMIENTOS}

Este estudio forma parte del Proyecto de Investigación EDU2009-12760, financiado por el Ministerio de Ciencia e Innovación (España), y del Proyecto de Excelencia P09-SEJ-5219, financiado por la Junta de Andalucía.

\section{REFERENCIAS BIBLIOGRÁFICAS}

Acevedo, J. A. (2004). Reflexiones sobre las finalidades de la enseñanza de las ciencias: educación científica para la ciudadanía. Revista Eureka sobre Enseñanza y Divulgación de las Ciencias, 1(1), pp. $3-16$. 
BAnet, E. (2010). El medio natural en la LOE: ¿continuidad o cambio en el currículo de educación primaria? Investigación en la Escuela, 70, pp. 71-78.

Cañal, P. (2006). La alfabetización científica en la infancia. Aula de Infantil, 33, pp. 5-9.

Castillo, S. y Cabrerizo, J. (2003). Evaluación educativa y promoción escolar. Madrid: Pearson.

Criado, A. M. y García-Carmona, A. (2011). Las experiencias prácticas para el conocimiento del medio (natural y tecnológico) en la formación inicial de maestros. Investigación en la Escuela, 74 , pp. 73-88.

De Pablo, P. (2006). CosmoCaixa. Espacio lúdico-científico en el que se aprende. Aula de Infantil, 33, pp. 10-15.

De Pro, A. y Miralles, P. (2009). El currículo de Conocimiento del Medio Natural, Social y Cultural en la Educación Primaria. Educatio Siglo XXI: Revista de la Facultad de Educación, 27(1), pp. 59-96.

De Pro, A.; Sánchez, G. y Valcárcel, M. V. (2008). Análisis de los libros de texto de Física y Química en el contexto de la Reforma LOGSE. Enseñanza de las Ciencias, 26(2), pp. 193-210.

ENCIENDE (2011). Informe: Enseñanza de las Ciencias en la Didáctica escolar para edades tempranas en España. Madrid: COSCE.

Fernández, J.; Elortegui, N.; Rodríguez, J. F. y Moreno, T. (2002, 2. a ed.). ¿Cómo hacer unidades didácticas innovadoras? Sevilla: Díada.

Furió Más, C. y Furió Gómez, C. (2009). ¿Cómo diseñar una secuencia de enseñanza de ciencias con una orientación socioconstructivista? Educación Química, 20(3), pp. 246-251.

García-Carmona, A.; Criado, A. M. y Cañal, P. (2014). ¿Qué educación científica se promueve para la etapa de Primaria en España? Un análisis de las prescripciones oficiales del currículo vigente. Enseñanza de las Ciencias, 32(1), 139-157

García De Cajén, S.; Domínguez, J. M. y García-Rodeja, E. (2002). Razonamiento y argumentación en ciencias. Diferentes puntos de vista en el currículo oficial. Enseñanza de las Ciencias, 20(2), pp. 217-228.

Garritz, A. (2009). La afectividad en la enseñanza de las ciencias. Educación Química, núm. extra, pp. 212-219.

Lera, M. J. (2006). Calidad de la Educación Infantil: Instrumentos de Evaluación. Revista de Educación, 343, pp. 301-323.

Marín, N. (2005). La enseñanza de las ciencias en educación infantil. Almería: Grupo Editorial Universitario.

Martín, E. (2008). Aprender a aprender: clave para el aprendizaje a lo largo de la vida. CEE Participación Educativa, 9, pp. 72-78

Osborne, J. y Dillon, J. (coord.) (2008). Science Education in Europe: Critical Reflections. London: Nuffield Foundation.

Padilla, M. T. (2002). Técnicas e Instrumentos para el Diagnóstico y la Evaluación Educativa. Madrid: CCS.

PaseK, E.; Matos, Y.; Villasmil, T. y Rojas, A. (2010). Los proyectos didácticos y la ciencia en Educación inicial. Acción Pedagógica, 19, pp. 134-144.

Pozo, J. I. y Gómez, M. A. (1998). Aprender y enseñar Ciencia. Madrid: Morata.

Pozuelos, F. J.; Travé, G. y Cañal, P. (2010). Inquiry-Based Teaching: Teachers Conceptions, Impediments and Support. Teaching Education, 21(2), pp. 131-142.

http://dx.doi.org/10.1080/10476210903494507

Prieto, T.; Blanco, A. y Brero, V. B. (2002). La progresión en el aprendizaje de dominios específicos: una propuesta para la investigación. Enseñanza de las Ciencias, 20(1), pp. 3-14.

Roás, J. M. (2001). El huerto escolar en la educación infantil. Aula de Innovación Educativa, 100, pp. 45-49. 
Rocard, M.; Csermely, P.; Jorde, D.; Lenzen, D.; Walberg, H. y Hemmo, V. (2007). Science Education Now: A Renewed Pedagogy for the Future of Europe. Brussels: Directorate General for Research, Science, Economy and Society.

Rodríguez, Y.; Patarroyo, L.; Sierra, L. M. y Arana, M. H. (2007). La educación científicotecnológica de educadores infantiles en la Universidad Pedagógica Nacional de Colombia. Tabula Rasa, 7, pp. 251-273.

Romero, G. (2011). Aprender, disfrutar, todos juntos por proyectos (el equilibrio). Aula de Infantil, 59, pp. 33-37.

Sánchez, M. A.; Gallegos, C.; Huerto, L. y Ribeiro, M. (2008). ¿Con qué saboreamos?: tareas y experiencias para un taller de ciencias. Revista Eureka sobre Enseñanza y Divulgación de las Ciencias, 5(2), pp. 200-211.

Spektor-Levy, O.; Kesner, Y. y Mevarech, Z. (2013). Science and Scientific Curiosity in Pre-school. The teacher's point of view. International Journal of Science Education, 35(13), pp. 2226-2253. http://dx.doi.org/10.1080/09500693.2011.631608

Tonucci, F. (1995). El niño y la ciencia. En: Con ojos de maestro, pp. 85-107. Buenos Aires: Troquel.

Varela, C. y Plasencia, I. C. (2006). El Proyecto Spectrum: aplicación y actividades de aprendizaje de ciencias en el primer ciclo de la Educación Primaria. Revista de Educación, 339, pp. 947-958. 


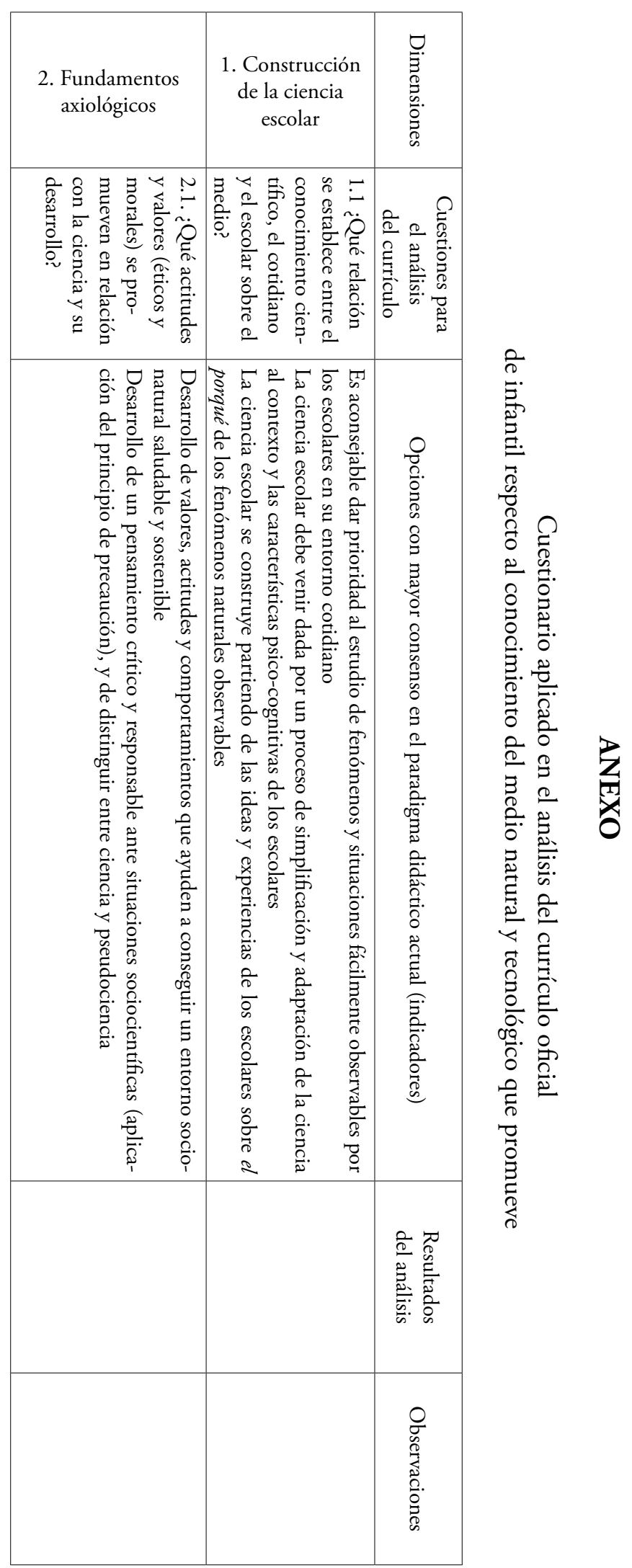




\begin{tabular}{|c|c|c|c|c|c|c|}
\hline 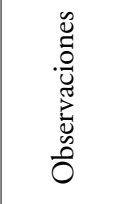 & & & & & & \\
\hline 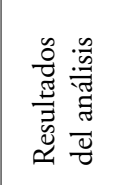 & & & & & & \\
\hline 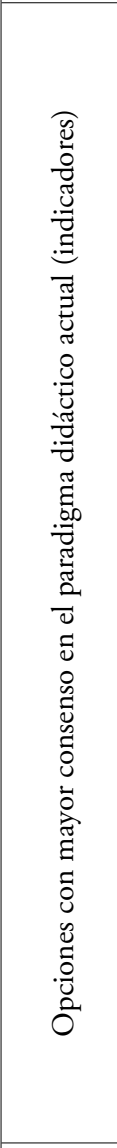 & 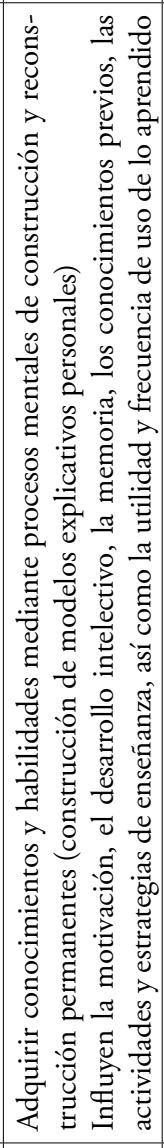 & 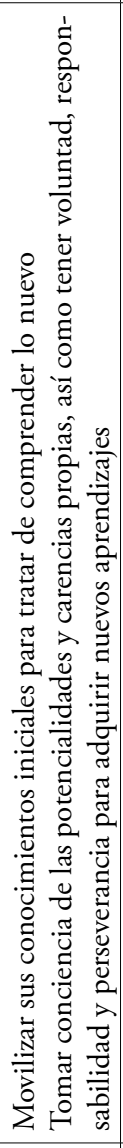 & 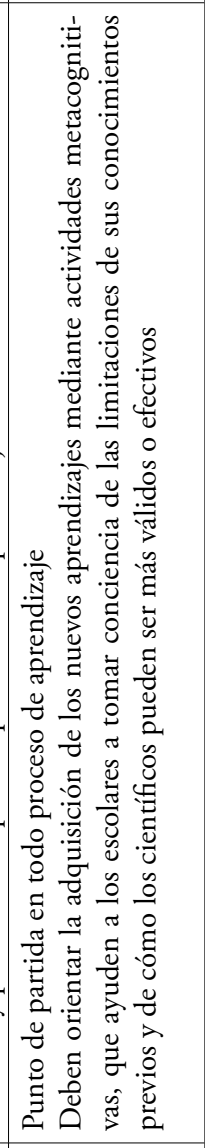 & 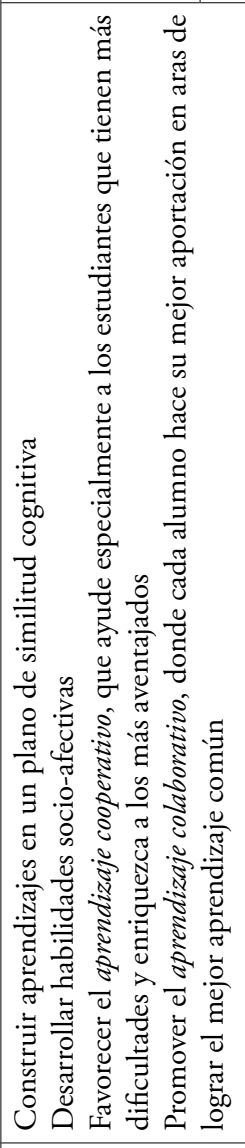 & 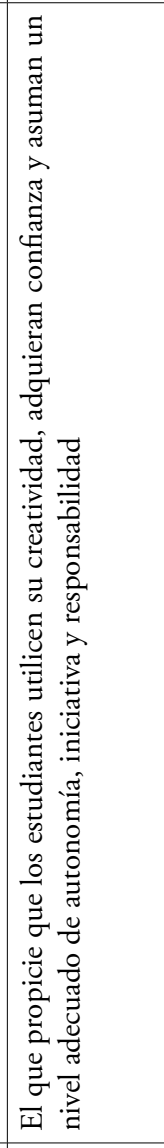 & 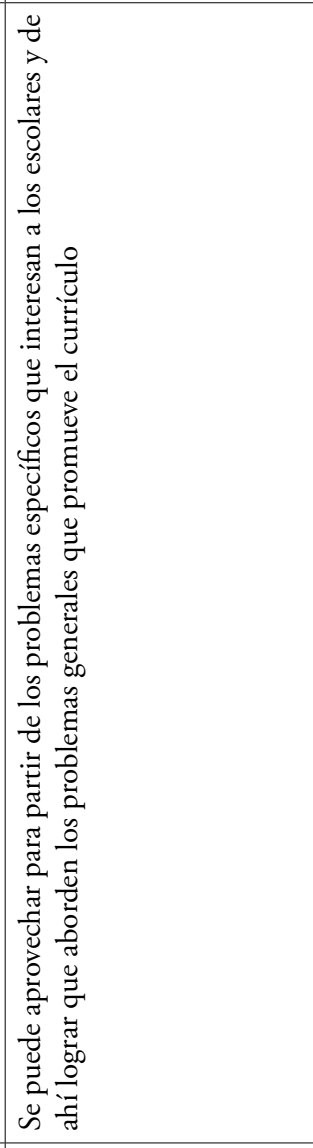 \\
\hline 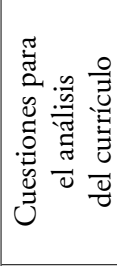 & 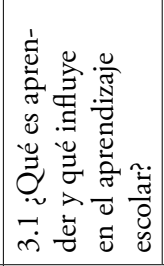 & 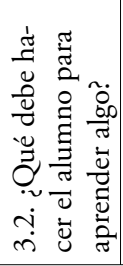 & 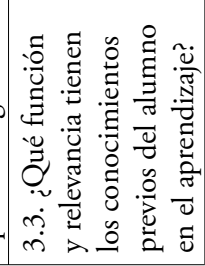 & 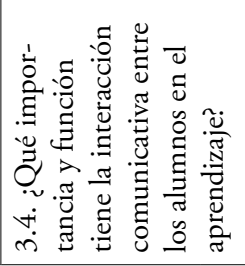 & 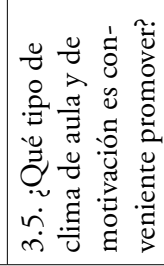 & 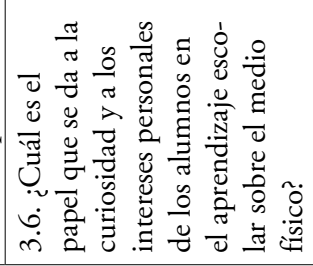 \\
\hline 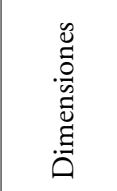 & \multicolumn{6}{|c|}{ soว!̊ogjoэ!sd soluəurepun $_{H}{ }^{\circ} \mathcal{E}$} \\
\hline
\end{tabular}




\begin{tabular}{|c|c|c|c|c|}
\hline \multicolumn{4}{|c|}{ 4. Objetivos y competencias } & \multirow{2}{*}{ 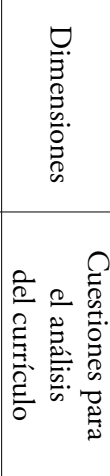 } \\
\hline 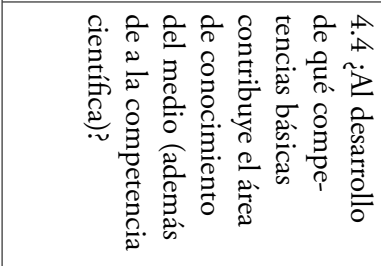 & 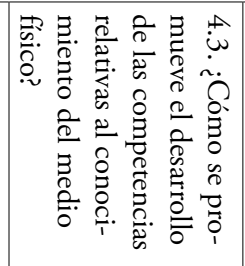 & 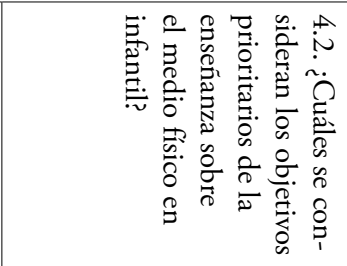 & 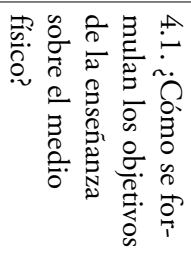 & \\
\hline 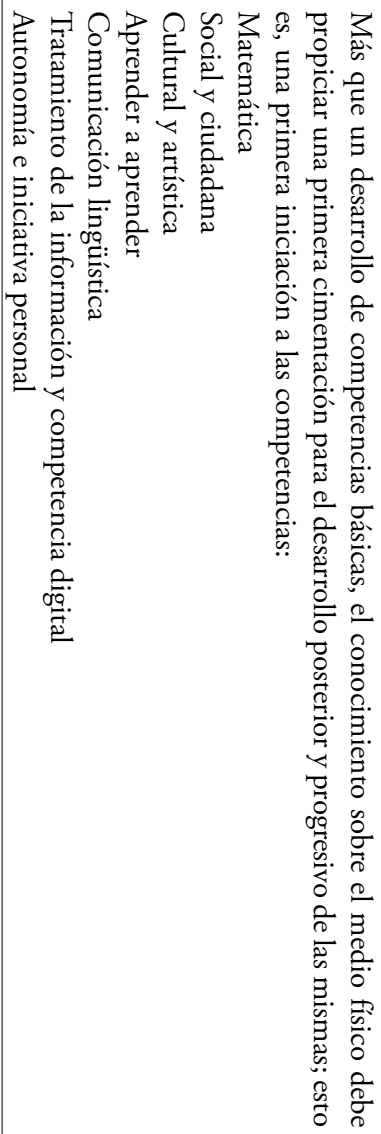 & 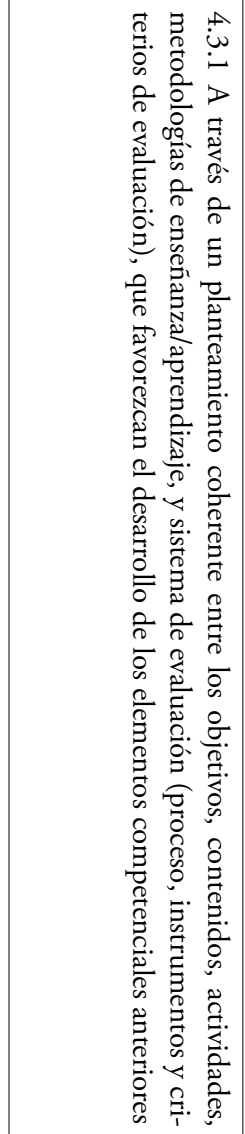 & 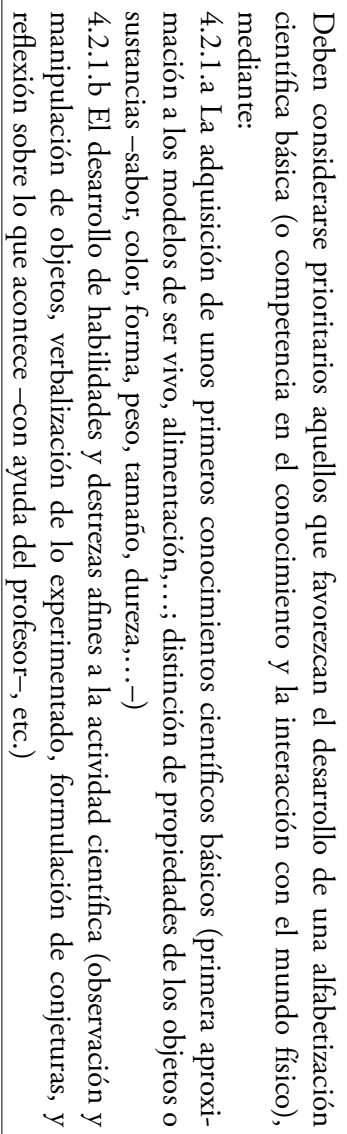 & 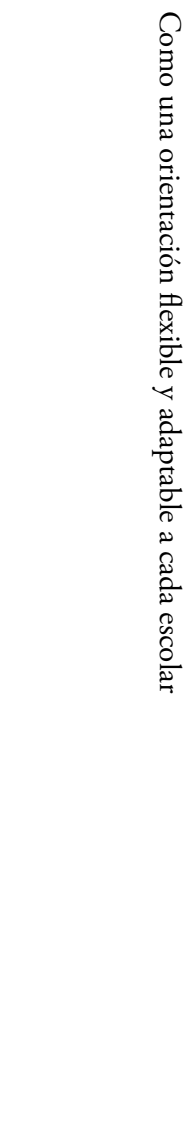 & 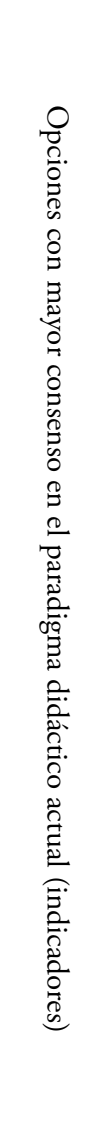 \\
\hline & & & & 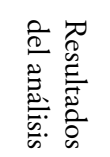 \\
\hline & & & & 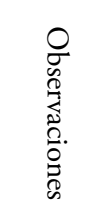 \\
\hline
\end{tabular}




\begin{tabular}{|c|c|c|c|c|c|c|c|}
\hline 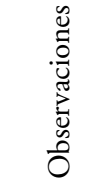 & & & & & & & \\
\hline 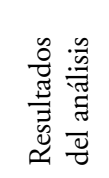 & & & & & & & \\
\hline 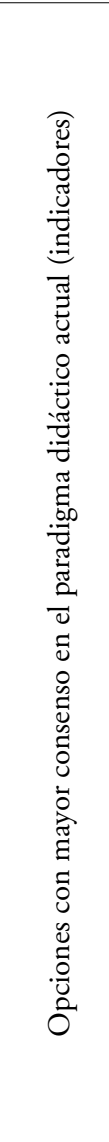 & 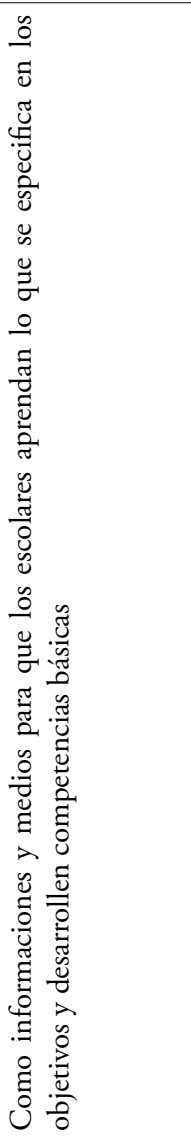 & 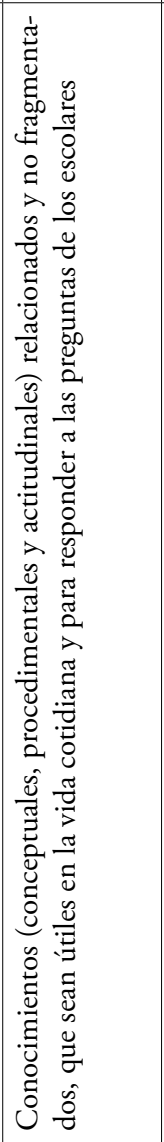 & 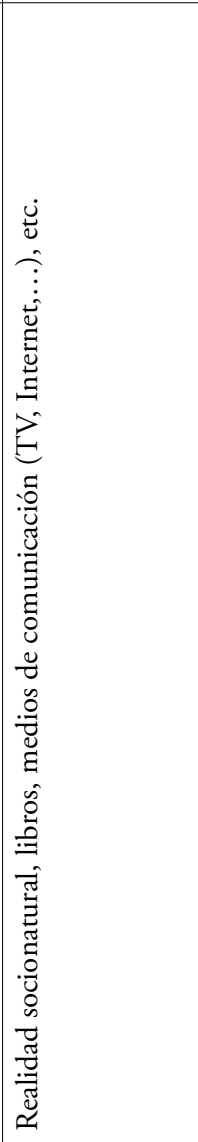 & 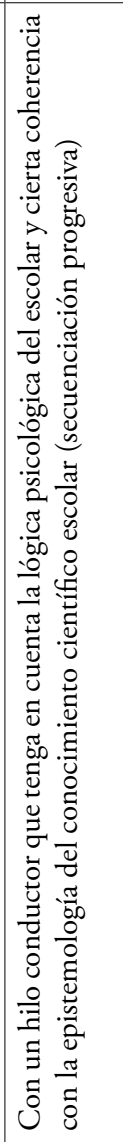 & 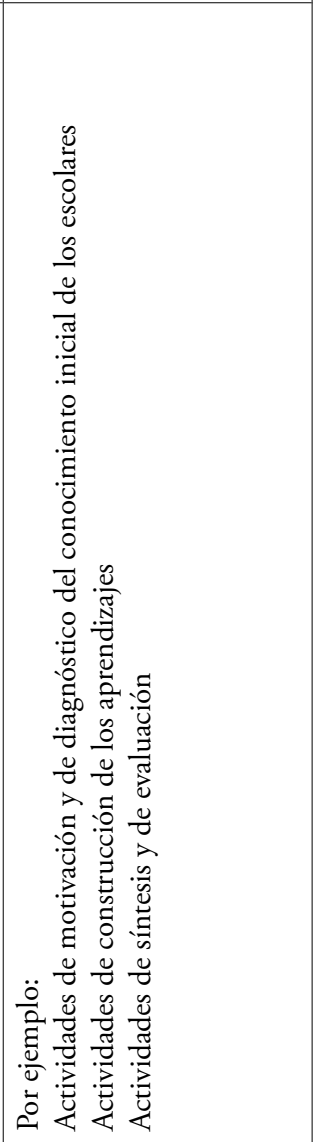 & 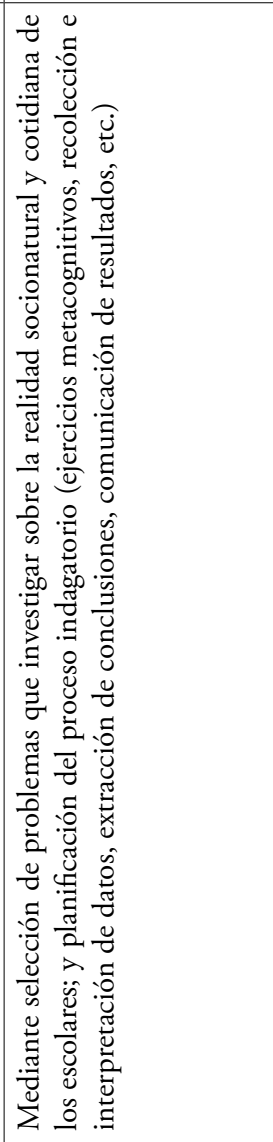 & 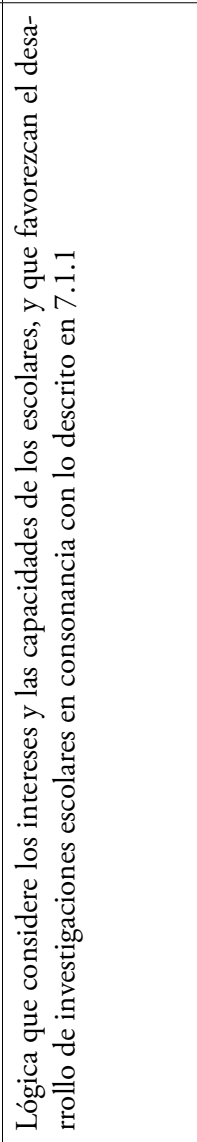 \\
\hline 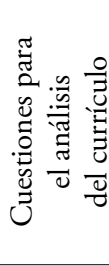 & 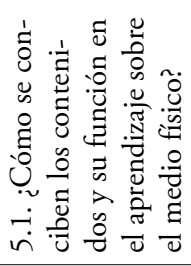 & 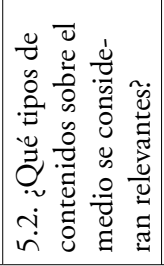 & 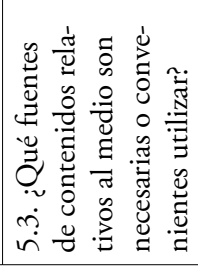 & 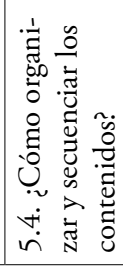 & 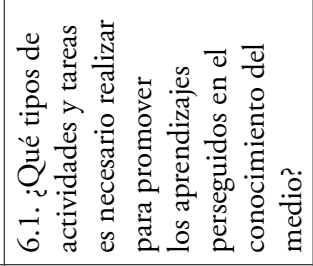 & 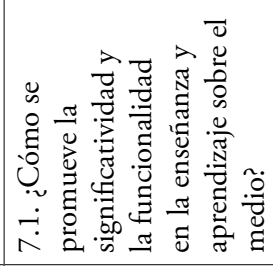 & 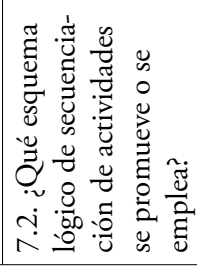 \\
\hline 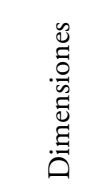 & \multicolumn{4}{|c|}{ 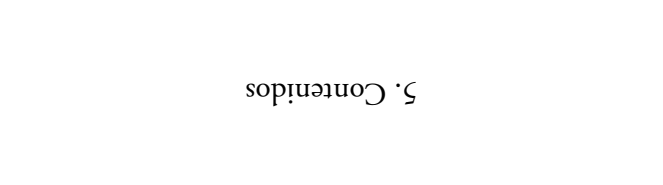 } & sәрер!м!ฺ७ 9 & \multicolumn{2}{|c|}{ 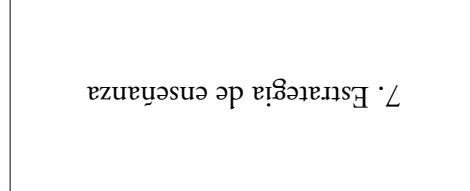 } \\
\hline
\end{tabular}




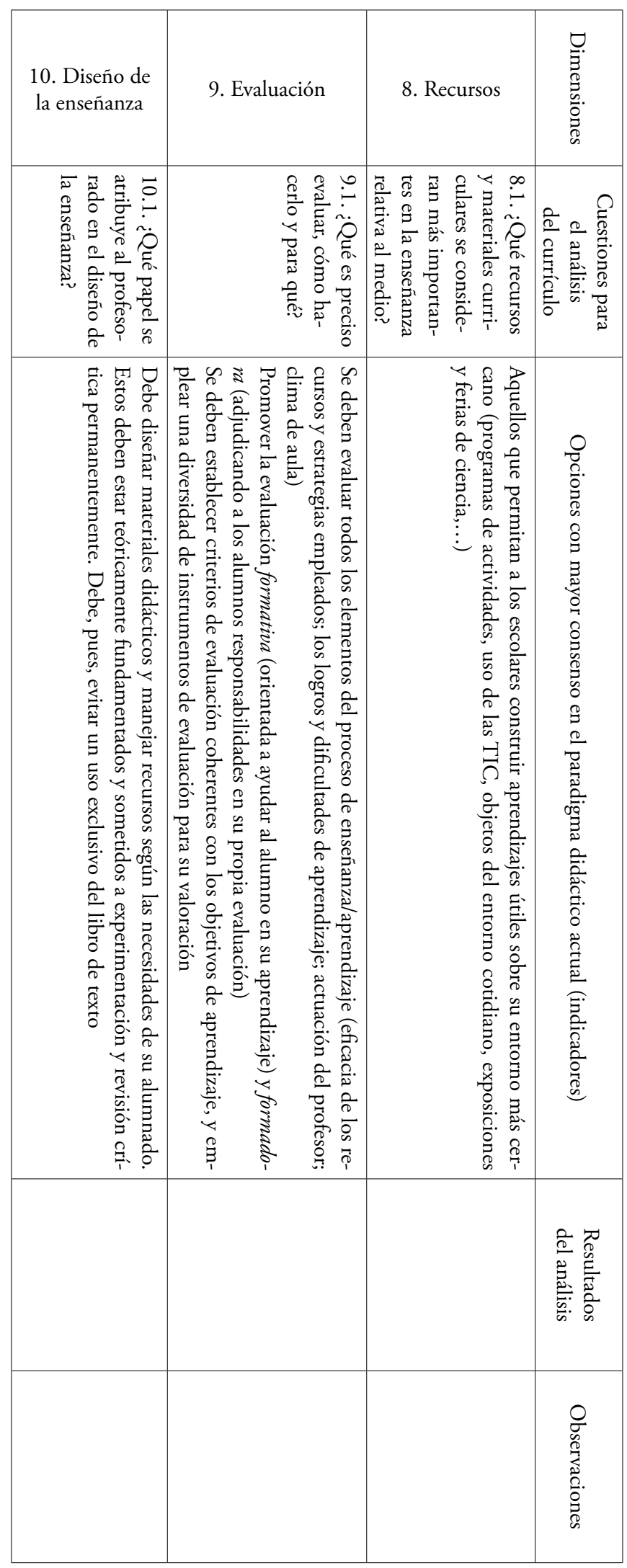




\title{
Scientific literacy at the 3-6 year old stage: an analysis of Spain's national curriculum
}

\author{
Antonio García-Carmona, Ana M. Criado, Pedro Cañal \\ Departamento de Didáctica de las Ciencias. Universidad de Sevilla \\ garcia-carmona@us.es, acriado@us.es, pcanal@us.es
}

An analysis is made of the scientific literacy promoted by Spain's national curriculum for the 3-6 year old stage. To this end, a questionnaire was designed and implemented combining inter- and intra-rater processes of analysis as a strategy of validation and reliability. An evaluation was made of how the official document addresses the following dimensions of science education at this stage: the construction of school science; the axiology and psychology of learning science; objectives and competences; content; teaching activities, design and strategies; teaching resources and evaluation. The results indicate that the school science actually being put forward in the document is, to a major degree, out of tune with current trends in Science Teaching. In view of the deficiencies identified in the document, the following improvements are recommended:

- There should be some insistence that pupils' ideas and prior experiences must constitute the starting point from which to guide the learning process, and that permanent communicative interaction in a context of cognitive similarity (under the teacher's guidance) fosters science learning.

- The document explicitly mentions the development of certain basic competences (communicative, mathematical, cultural and digital) in the area of environmental knowledge. It would be advisable, however, to present a firmer commitment to science education based on the development of competences. In particular, special attention should be paid to the pupils' competences in socialization and affect, since these are extensively accepted in the current literature as essential to achieving better basic science education. In this regard, advantage should be taken of the opportunities offered by recreational science workshops that generate positive emotions while the pupils are learning and by workshops constructing simple devices aimed at providing an initiation into technological thinking, in a socio-affective and game-like climate. Similarly, attention should be paid to the competence of learning-to-learn by fostering pupils' curiosity, keenness, patience and perseverance as essential attitudes for a first knowledge of the nature of the world that surrounds them.

- It would be advisable to provide some guidance, both for the introduction and progressive development of school science knowledge over the course of the stage being considered, and for the corresponding evaluation criteria.

- Attention should be paid to teaching and learning activities, offering some basic guidance on their design, sequencing, educational purposes, etc.

- Since a research-based strategy of science learning is being promoted, there should be appropriate treatment of the essential features of this teaching model and of the possible approaches to its implementation.

- Beyond merely citing the environment as a setting in which to initiate science learning at school, there should be explicit reference to a variety of specific resources that foster its development both in the classroom (e.g., corners of the room devoted to science) and outside it (scientific exhibits, gardens,...).

- Teachers should be encouraged to design or adapt their own teaching materials, promoting not only the teaching resources and methodological approaches of the point just mentioned above, but also the progressive integration of materials and tools normally used by society to understand the natural world (scales, sensors, optical instruments, documentaries, books, computers,...).

- Attention should be paid to the evaluation's formative dimension, in which some of the responsibility of evaluation is allocated to the pupils themselves.

- There should be some allusion made to a variety of tools for evaluation, not just direct observation in the classroom.

The inclusion of suggestions such as those above in the official document under review will not by itself mean that they will be taken up by teachers in their everyday practice. Nevertheless, unless they are integrated into framework documents like the present one, they may not be regarded as essential, and therefore their impact on classroom practice is likely to be imperceptible. 